\title{
Rechtsgutachten zur Neugestaltung der Datenschutzkontrolle in Hessen
}

\section{Beitrag zur Klärung der Konsequenzen aus dem Urteil des EuGH vom 9. März 2010}

\section{Die Situation in Hessen, Handlungsbedarf}

Das Land Hessen ist verpflichtet, den Verstoß gegen das EU-Recht zu beheben, wenn die hessische Datenschutzkontrolle nicht in „völliger Unabhängigkeit" erfolgt. Dabei ist zu berücksichtigen, dass in Hessen die Datenschutzkontrolle für den öffentlichen und den privaten Bereich getrennt ist. Handlungsbedarf besteht, wenn in beiden Bereichen oder auch nur einem Bereich ein Verstoß gegen EU-Recht festzustellen ist.

Nach $₫ 22$ HDSG ist der HDSB als oberste Landesbehörde in Ausübung seines Amtes unabhängig und nur dem Gesetz unterworfen. Für den öffentlichen Bereich besteht - isoliert betrachtet - keine Handlungsnotwendigkeit.

Das Regierungspräsidium Darmstadt überprüft als Aufsichtsbehörde nach $\$ 38$ Abs. 1 BDSG die Ausführung des Bundesdatenschutzgesetzes sowie anderer Vorschriften über den Datenschutz in Hessen, soweit diese die automatisierte Verarbeitung personenbezogener Daten oder die Verarbeitung oder Nutzung personenbezogener Daten in oder aus nicht automatisierten Dateien regeln. Es unterliegt als staatliche Mittelbehörde der Fachaufsicht und Rechtsaufsicht des Hessischen Innenministeriums. Damit ist es im Sinne der Rechtsprechung des EuGH nicht „völlig unabhängig“, d.h. die Ausgestaltung der Datenschutzkontrolle widerspricht eindeutig den Anforderungen des EuGH. Es besteht Handlungsbedarf.

\section{Zum Rechtsgutachten}

Das auf Bitte des Hessischen Landtags vom Hessischen Datenschutzbeauftragten erstattete Rechtsgutachten zur Neugestaltung der Datenschutzkontrolle und zur Verfassungsmäßigkeit einer Zusammenlegung des privaten und öffentlichen Bereichs der Datenschutzkontrolle in Hessen unter Zugrundlegung des Gesetzentwurfs der Fraktion der SPD (Drucksache 18/375) betrifft die Frage, in welchem Umfang die vom EuGH in seinem Urteil vom 9. März 2010 (C 518/07) geforderte völlige Unabhängigkeit der Datenschutzkontrolle im Einklang mit den fundamentalen Datenschutzprinzipien des Grundgesetzes hergestellt werden kann. Ausgehend von dem Befund, dass die Datenschutzkontrolle zwei Bereich abdeckt, nämlich die Kontrolle der Verwaltung (öffentlicher Bereich) und die Kontrolle durch die Verwaltung (nicht-öffentlicher Bereich) stellt sich die Frage, ob die beiden Bereiche verknüpft werden können. Die bisherige Verknüpfung beider Bereiche in der Bundesrepublik Deutschland hat nach Ansicht des EuGH dazu geführt, dass der Datenschutz im nicht-öffentlichen Bereich nicht völlig unabhängig ist. Die Konsequenz ist die strikte Trennung beider Bereiche oder die Suche nach neuen Verbindungsmöglichkeiten. Gegen die Trennung spricht, dass sich öffentlicher und privater Bereich gar nicht mehr unterscheiden lassen. Die Verknüpfung wiederum bringt die Schwierigkeit mit sich, dass das deutsche Demokratieprinzip grundsätzlich eine Ministerialverantwortlichkeit erfordert. Nach Europarecht und nationalem Verfassungsrecht muss der einheitliche Datenschutz dementsprechend weiterhin völlig unabhängig sein, eine permanente effektive parlamentarische Kontrolle ermöglichen, einen effektiven Schutz der Datenschutzgrundrechte als Abwehr-, Teilhabe- und Leistungsgrundrechte gewährleisten.
Das Gutachten zeigt auf, wie dies zu bewerkstelligen ist. Erforderlich ist zunächst eine Ausweitung der parlamentarischen Verantwortlichkeit des Datenschutzbeauftragten, die aber nicht zu einer parlamentarischen Parallelverwaltung führen darf. Vielmehr ist die sachliche Unabhängigkeit des Datenschutzbeauftragten, auch gegenüber dem Parlament, zu wahren. Auch eine Fach- und Rechtsaufsicht des Parlaments kommt nicht in Betracht, wohl aber eine Dienstaufsicht des Landtagspräsidenten über den Hessischen Datenschutzbeauftragten. Die Kontrollschutzkontrolle im privaten Bereich erfordert keine Eingriffsbefugnisse, die zwar de lege lata gegeben sind, jedoch im Zuge der anstehenden Runde um Erneuerung des Bundesdatenschutzgesetzes und Hessischen Datenschutzgesetzes optimiert werden sollten. Das Problem der Ministerverantwortlichkeit lässt sich lösen. Die Ministerverantwortlichkeit gilt nicht ausnahmslos. Auch das BVerfG hat in Einzelfällen Ausnahme zugelassen. Die Ministerverantwortlichkeit ist somit nicht conditio sine qua non des Demokratieprinzips. Ihre ausnahmslose Durchsetzung ist nicht so fundamental, dass die Bundesrepublik sich unter Berufung auf den Souveränitätsvorbehalt über die Interpretation der Europäischen Richtlinien durch den EuGH hinwegsetzen könnte und dürfte. Das Gutachten kann in Kürze auf der Homepage des Hessischen Datenschutzbeauftragten $w w w$.datenschutz.hessen.del abgerufen werden.

Prof. Dr. Michael Ronellenfitsch Der Hessische Datenschutzbeauftragte 